\title{
Self management of patients with mild COPD in primary care: randomised controlled trial
}

\author{
Kate Jolly, ${ }^{1}$ Manbinder S Sidhu, ${ }^{2}$ Catherine A Hewitt, ${ }^{3}$ Peter A Coventry, ${ }^{4}$ Amanda Daley, ${ }^{5}$ \\ Rachel Jordan, ${ }^{1}$ Carl Heneghan, ${ }^{6}$ Sally Singh, ${ }^{7}$ Natalie Ives, ${ }^{3}$ Peymane Adab, ${ }^{1}$ Susan Jowett, ${ }^{1,8}$ \\ Jinu Varghese, ${ }^{9}$ David Nunan, ${ }^{6}$ Khaled Ahmed, ${ }^{1}$ Lee Dowson, ${ }^{10}$ David Fitzmaurice ${ }^{11}$
}

For numbered affiliations see end of the article

Correspondence to: K Jolly c.b.jolly@bham.ac.uk

Additional material is published online only. To view please visit the journal online.

Cite this as: BMJ 2018;361:k2241 http://dx.doi.org/10.1136/bmj.k2241

Accepted: 17 April 2018

\section{Abstract}

\section{Objective}

To evaluate the effectiveness of telephone health coaching delivered by a nurse to support self management in a primary care population with mild symptoms of chronic obstructive pulmonary disease (COPD).

\section{Design}

Multicentre randomised controlled trial.

Setting

71 general practices in four areas of England.

Participants

577 patients with Medical Research Council dyspnoea scale scores of 1 or 2 , recruited from primary care COPD registers with spirometry confirmed diagnosis. Patients were randomised to telephone health coaching $(n=289)$ or usual care $(n=288)$.

Interventions

Telephone health coaching intervention delivered by nurses, underpinned by Social Cognitive Theory. The coaching promoted accessing smoking cessation services, increasing physical activity, medication management, and action planning (4 sessions over 11 weeks; postal information at weeks 16 and 24). The nurses received two days of training. The usual care group received a leaflet about COPD.

Main outcome measures

The primary outcome was health related quality of life at 12 months using the short version of the St George's Respiratory Questionnaire (SGRQ-C).

\section{WHAT IS ALREADY KNOWN ON THIS TOPIC}

Current policy for the prevention and management of long term conditions focusses on efforts to prevent onset or slow progression of disease early in the disease trajectory

This prevention paradigm has only recently been adopted for chronic obstructive pulmonary disease (COPD)

Systematic reviews have shown that self management support for patients with COPD is effective in improving health related quality of life and in reducing hospital admissions, but the evidence comes largely from patients with moderate or severe disease and predominantly recruited from secondary care

\section{WHAT THIS STUDY ADDS}

Telephone health coaching comprising components that were theoretically associated with slowing decline of lung function, did improve self management activities that were targeted by the intervention in patients with mildly symptomatic COPD recruited from primary care Health related quality of life was not improved over the 12 month follow-up period
Results

The intervention was delivered with good fidelity: $86 \%$ of scheduled calls were delivered; $75 \%$ of patients received all four calls. $92 \%$ of patients were followedup at six months and $89 \%$ at 12 months. There was no difference in SGRQ-C total score at 12 months (mean difference $-1.3,95 \%$ confidence interval -3.6 to 0.9 , $\mathrm{P}=0.23)$. Compared with patients in the usual care group, at six months follow-up, the intervention group reported greater physical activity, more had received a care plan ( $44 \% \vee 30 \%$ ), rescue packs of antibiotics (37\% v 29\%), and inhaler use technique check (68\% v 55\%).

\section{Conclusions}

A new telephone health coaching intervention to promote behaviour change in primary care patients with mild symptoms of dyspnoea did lead to changes in self management activities, but did not improve health related quality of life.

Trial registration

Current controlled trials ISRCTN 06710391

\section{Introduction}

Chronic diseases, such as chronic obstructive pulmonary disease (COPD), are a major cause of death and disability in high income countries and of rising importance in low and middle income countries. ${ }^{1}$ Owing to their high prevalence and chronicity, current international policy focuses on the need to support patients to self manage their conditions. ${ }^{2}$ Most interventions designed to support self management have been targeted at people with more severe disease who are likely to be motivated to change behaviour, and where there is the most opportunity for symptomatic improvement. However, more recent efforts have aimed to prevent onset or slow progression early in the disease course to reduce the burden and costs of treating more advanced disease later. This prevention model has only recently been adopted in COPD, with calls for interventions to reduce risk in people with early disease. ${ }^{3}$

The growing number of people at risk of developing long term conditions and the prevalence of early disease, means an accessibleand low resourceapproach needs to be taken to support self management. One such approach is to use interactive telephone health coaching, with the coach and patient working together to identify barriers to behaviour change and finding ways to overcome them. Key techniques include modelling behaviour, goal setting, and empowering the patient to improve their health status. ${ }^{4}$ Telephone health coaching has shown potential benefits on self 
efficacy, health behaviour, and health status in a rapid review of trials in long term conditions. ${ }^{5}$

COPD is a common respiratory condition with an estimated 65 million people worldwide with moderate or severe disease. ${ }^{1}$ Like most chronic diseases, it causes a considerable burden on health services and society and is a leading cause of death in most countries. ${ }^{67}$ Interventions to support self management in patients with COPD have been shown to be effective in improving health related quality of life and in reducing hospital admissions among patients with COPD, ${ }^{89}$ but trials have largely recruited people from secondary care and excluded those with mild disease. ${ }^{9}$ However, patients with mild dyspnoea represent $38 \%$ to $54 \%$ of diagnosed patients in primary care. ${ }^{1011}$ This is likely to increase with case finding initiatives to identify disease in people with symptoms. ${ }^{12}$

Many components of self management interventions could promote better health and prevent disease progression in the early stages of COPD. Smoking is a major cause of COPD, and smoking cessation has been shown to be beneficial in maintaining better lung function and in slowing disease progression across all severity levels. ${ }^{13}{ }^{14}$ Reduced physical activity level is an independent risk factor for exacerbations, hospital stays, and mortality among those with COPD and occurs even in the early stages of disease. ${ }^{15-17}$ Inhaler treatments have well established efficacy in reducing exacerbations and admissions among patients with moderate and severe COPD, and growing evidence of efficacy in improving clinical outcomes and reducing decline in lung function among patients with more mild impairment. ${ }^{1819}$ Any intervention that improves medication adherence and inhaler use technique, which is frequently poor, ${ }^{20}$ is thus likely to improve outcomes for patients. Sixty per cent of primary care patients with COPD report exacerbations of their disease, ${ }^{10}$ which are associated with more rapid decline in lung function. ${ }^{14}$ Interventions that aim to reduce the severity of exacerbations include prompting early recognition of symptoms and rapid use of antibiotics or corticosteroids, or both, either through seeking a primary care appointment or use of a self treatment rescue drug pack.

We evaluated telephone health coaching in patients with mildly symptomatic COPD to explore the effectiveness of supporting self management activities in this group of patients. We hypothesised that a telephone health coaching intervention delivered by a nurse to support self management, compared with usual primary care, would lead to improved COPD health related quality of life at 12 months followup and would improve physical activity, smoking cessation, self management behaviours, psychological health, and self efficacy.

\section{Methods}

Design

Patient self management for chronic obstructive pulmonary disease (COPD) was a pragmatic multicentre phase III randomised controlled trial
(RCT) of a telephone health coaching intervention to support self management compared with usual care for people with COPD with mild dyspnoea. Details of the study protocol have been published elsewhere. ${ }^{21}$ We followed the CONSORT guidelines for reporting RCTs of non-pharmacological treatments to report this study. ${ }^{22}$ After publication of the protocol in the ISRCTN clinical trial registry at the feasibility study phase, we changed the inclusion criterion for post-bronchodilator spirometry from below the lower limit of normal to forced expiratory volume in one second/forced vital capacity $<0.7$, which is that recommended by the Global Initiative for Obstructive Lung Disease (GOLD). ${ }^{6}$ We also included some additional subgroup analyses to those in the published protocol (baseline forced expiratory volume in one second predicted $(\geq 80$ or $<80$ ) and degree of limitation of activities in the St George's Respiratory Questionnaire (SGRQ-C)). ${ }^{21}$ We embedded a substudy that investigated participant recruitment materials. In this substudy, general practices were randomised to send out either the standard participant information leaflet or a participant information leaflet which contained an additional web address and Quick Response code to give access to web-based materials including podcasts about taking part in research in general and in the patient self management COPD trial in particular. ${ }^{23}$ This did not alter any other trial processes.

\section{Participants}

Participants were recruited from 71 general practices within England located in Birmingham and West Midlands South, Greater Manchester, West Midlands North, and Oxfordshire or Gloucestershire. Patients aged over 18 were identified as eligible if they were on the practice COPD register, thus had respiratory symptoms consistent with COPD; reported mild dyspnoea (MRC grades 1 (only breathless on strenuous exercise) or 2 (only get short of breath when hurrying on level ground or up a slight hill)) at the baseline assessment; had a forced expiratory volume in one second/forced vital capacity $<0.7$ after postbronchodilator spirometry (consistent with current UK guidelines) ${ }^{24}$ at the baseline assessment. If there was a contraindication or the patient was unable to perform or refused spirometry, a spirometry result reported from hospital within the last 18 months was used. Doctors were asked to exclude patients who they considered to be inappropriate for the study (eg, had a terminal disease or a severe psychiatric disorder). Eligible patients were sent a letter of invitation, information brochure, and information leaflet from their practice, with a reply slip to the research team which included the MRC dyspnoea scale. Patients with MRC grade 1 or 2 , and those without a recorded dyspnoea score were invited to participate.

\section{Baseline assessment}

Patients who expressed an interest in the study were telephoned by a researcher and invited to a recruitment assessment at their practice, undertaken by a research 
nurse or trained researcher. Patients who attended baseline assessments were given the opportunity to discuss the study. After informed consent, postbronchodilator spirometry was undertaken, height and weight were measured, and the patient was asked to complete a baseline questionnaire pack. This questionnaire pack included questions on patient demographics and the measures for the primary and secondary outcomes. A GENEActiv accelerometer was fitted on their non-dominant wrist, which they were asked to return by post in a prepaid envelope after seven days of continuous wear.

\section{Intervention and usual care}

This was a pragmatic trial with no constraints on doctors' management of the participants in either group.

The usual care group received a standard information leaflet about self management of COPD. ${ }^{25}$ The 13 page leaflet gave a definition of COPD, a detailed description of associated symptoms, how the illness can be managed with the use of inhalers, how to treat exacerbations, and details of other resources (eg, British Lung Foundation and NHS Smokefree).

The intervention consisted of telephone health coaching delivered by a nurse with supporting written documents, a pedometer, and a self monitoring diary. This aimed to support self management in relation to smoking cessation, physical activity increases, correct inhaler use technique, and medication adherence. For those with recurrent exacerbations, it also aimed to improve patient confidence in identifying an exacerbation early in order to start rescue drugs (ie, antibiotics or steroids).

Social Cognitive Theory underpinned the intervention, ${ }^{26}$ and included education, monitoring, and assessment of progress, and taught skills with the aim of increasing self efficacy. ${ }^{27} 28$ We incorporated best evidence for the promotion of physical activity (tailored, ongoing support, duration six months, use of pedometer). ${ }^{29-32}$ The intervention components are detailed in web appendix 1 . The first telephone coaching session at one week after randomisation aimed to last 35-60 minutes (determined by the number of issues requiring discussion, such as current smoking), followed by a 15-20 minute telephone session at weeks 3,7 , and 11 with written supportive materials tailored to the patient after each telephone call (eg, summary of goals agreed, physical activity diary, contact details for local services, information leaflet showing correct inhaler use technique). Nurses provided standard written prompts or information at weeks 16 and 24.

The eight nurses attended two days of training and practiced telephone coaching sessions with the research team. Nurses followed a proforma to guide the consultation in accordance with the telephone consultation protocol. The nurses briefly summarised the content of the call and any actions agreed after each telephone call. A sample of telephone consultations were recorded with the patients consent and reviewed by one researcher to determine compliance with the content of the intervention.

\section{Randomisation and masking}

Patients who had given informed consent and completed all the baseline measures were individually randomised in a 1:1 ratio to the telephone health coaching or usual care group stratified by centre. The allocation was made using a web-based programme hosted by the Primary Care Clinical Research and Trials Unit, University of Birmingham. Centre specific randomisation lists were produced by a statistician at the trials unit. The four recruitment centres were Birmingham and West Midlands South, Greater Manchester, West Midlands North, and Oxfordshire or Gloucestershire. Only the Primary Care Clinical Research and Trials Unit had access to the allocation sequence. Patients were informed of their allocation at the end of the recruitment appointment; they were not masked to treatment allocation. Data were entered into the study database by researchers at the University of Birmingham who were masked to the treatment allocation.

\section{Outcome assessment}

We measured outcomes by postal questionnaire at six months to determine short term change to the end of the intervention and at 12 months to determine whether any change was sustained. At 12 months, accelerometers were posted to participants with a follow-up telephone call to explain how to start the recording. They were asked to wear the accelerometers continuously for seven days and then to return them by post. Non-responders were telephoned and given the option of completing the questionnaire over the telephone.

\section{Outcomes}

The primary outcome measure was health related quality of life at 12 months from randomisation measured using the SGRQ-C. ${ }^{33}$ Scores range from 0 to 100 , with higher scores indicating greater impairment of quality of life.

Secondary outcomes were the MRC dyspnoea scale, ${ }^{34}$ self reported physical activity (using the International Physical Activity Questionnaire), ${ }^{35}$ psychological morbidity (using the Hospital Anxiety and Depression Scale), ${ }^{36}$ self efficacy for managing their COPD and undertaking physical activity (using the Stanford self efficacy scale), ${ }^{28}$ and health state utility (using the EuroQoL 5 Dimensions 5 Levels) ${ }^{37}$ at six and 12 months from randomisation. Health related quality of life at six months from randomisation measured using the SGRQ-C was also a secondary outcome. Smoking cessation rates and physical activity measured with GENEActiv accelerometers were assessed at 12 months. Prespecified exploratory outcomes were self management activities (related to smoking cessation, medication adherence, care plans, etc) reported by the patients and healthcare use at six and 12 months. An economic evaluation has also been undertaken, but will be reported elsewhere. 
Adverse events were reported by intervention participants during telephone calls and from the six and 12 month follow-up questionnaires; they were independently assessed by two independent clinicians.

\section{Statistical justification for sample size}

The sample size was determined to detect a difference in the SGRQ-C at 12 months. To have $80 \%$ power to detect a difference of four points (the minimal clinically significant difference) ${ }^{38}$ from a baseline total score value of $39,{ }^{39}$ with a standard deviation of 15 at the $5 \%$ level of significance required data from 445 patients. Allowing for an attrition rate of $20 \%$ at 12 months, we needed 556 participants (278 for each group).

The power to detect differences in self reported physical activity and in smoking cessation rates are detailed in the protocol paper. ${ }^{21}$

\section{Analysis}

All data were analysed by intention to treat. The main analyses compared primary and secondary outcome measures between treatment groups at 12 months after randomisation to assess the long term effect of the self management intervention. Data were also analysed at six months to assess the short term change.

The primary outcome and other continuous secondary outcome measures were analysed using a linear regression model. Ordered categorical secondary outcome measures (eg, MRC dyspnoea scale) were analysed using an ordinal logistic regression model. All primary and secondary analyses were adjusted for baseline values and centre. Differences between treatment groups were summarised using suitable effect estimates (eg, mean difference and odds ratio) with $95 \%$ confidence intervals. We used a 5\% statistical significance level.

Exploratory outcome measures were not analysed using statistical modelling except for the count data. Binary or categorical outcome measures were analysed using $\chi^{2}$ or Fisher's exact test and continuous measures were analysed using t-tests or a non-parametric equivalent (eg, Wilcoxon rank test). Measures of count were analysed using a Poisson regression model or negative binomial model as appropriate to obtain an incidence rate ratio. Models included an adjustment for baseline values and centre and an offset term for length of follow-up.

Several sensitivity analyses were performed for the SGRQ-C. Firstly, a per-protocol analysis which included only those patients who received all four telephone calls in the intervention group and excluded the one patient in the usual care group who received the intervention by mistake. Secondly, an analysis to assess the effect of missing data, with patients with missing 12 month SGRQ-C scores being simulated by regression imputation using baseline data, with baseline score, age, sex, MRC score, and treatment group used as predictors to impute missing scores. All participants were included in this analysis unless they had died by 12 months or both the baseline and
12 month SGRQ-C scores were missing. Finally, an analysis which excluded participants where the 12 month SGRQ-C questionnaires were returned either early ( $>1$ month before the assessment due date) or late ( $>65$ days after the assessment due date).

Subgroup analyses to explore the effects of the intervention in different patient subgroups were undertaken for the primary outcome. The subgroups, prespecified in the statistical analysis plan included participant characteristics (age, sex, ethnicity, smoking status, baseline MRC dyspnoea score, and number of comorbidities), active engagers with the intervention (through increased physical activity, uptake of smoking cessation support, or checking of inhaler use technique), baseline level of physical activity (from both the International Physical Activity Questionnaire and the accelerometer data), baseline health related quality of life (SGRQ-C), baseline self efficacy (Stanford), and baseline depression and anxiety (from the Hospital Anxiety and Depression Scale). Two post hoc subgroup analyses were also undertaken for baseline forced expiratory volume in one second predicted ( $\geq 80$ or $<80$ ) and degree of limitation of activities in the SGRQ-C. A treatment group by subgroup interaction parameter was included in the linear regression model to assess whether there were any differences in the treatment effect across the different strata. Differences between treatment groups within subgroups were only examined if the interaction parameters were statistically significant $(\mathrm{P}<0.05)$.

Details of the accelerometery analyses and available accelerometry data are provided in web appendix 2 .

\section{Patient involvement}

The study was supported by a COPD patient advisory group which provided input to a programme of research on COPD. The group met on a regular basis and one was a member of the trial management group for the duration of the study. The group commented on the initial design of the study, the burden of the trial assessment process, participant facing materials, and on the content and material to support the intervention. Additionally, the Trial Steering Group had a lay member. At the end of the study, the group commented on the findings and contributed to the dissemination plan.

\section{Results}

We sent a screening questionnaire and invitation leaflet to 5279 people on the COPD registers of 71 general practices; 2066 responded with an interest in the study, but 920 were excluded as they had an MRC dyspnoea scale score of 3 or more. Figure 1 shows that we screened 728 people at their practice between 18 March 2014 and 5 February 2015; 577 were eligible and randomised to telephone health coaching $(n=289)$ or usual care $(n=288)$. In total, 531 (92\%) of participants provided data at six months and $516(89 \%)$ at 12 months follow-up. There was imbalance in the follow-up rates between telephone health coaching $(82.7 \%$; 37 withdrawals) and usual 
care (96.2\%; 7 withdrawals) groups at 12 months, largely owing to patients who wished to withdraw from telephone health coaching also withdrawing from further follow-up. Of the 37 patients who withdrew from the telephone health coaching group, four withdrew before receiving any intervention and 16 withdrew during the intervention; eight cited illness and 10 cited intervention related factors ranging from it being too demanding to insufficiently so. Seventeen participants withdrew after the six months follow-up. Patients who withdrew from the study did not differ in characteristics to the full sample.

Table 1 shows the patient characteristics. Participants were predominantly white; $63 \%$ were male; the mean age was $70.4 ; 23 \%$ were current smokers; and only $19 \%$ were in paid employment. Participants had mild disease: mean FEV1 was 71.6\% predicted, 193 (33\%) were GOLD stage 1 and 309 (54\%) GOLD stage 2; 165 (28.6\%) reported MRC level I dyspnoea and 270 (47\%) reported medication for an exacerbation in the previous 12 months. The mean SGRQ-C total score was
28.7. The study groups were generally well balanced in terms of patient characteristics, although there was a higher proportion of current smokers in the telephone health coaching group. The usual care group reported a higher level of self reported moderate and vigorous physical activity, but this was not reflected in the accelerometry data at baseline. The accelerometry data for all participants showed that participants did a median of 31 minutes of moderate or vigorous activity in bouts of at least 10 minutes daily (interquartile range $0-160$ ). Participants who did not provide data at 12 months were more likely to be in GOLD stage 3, to be smokers, had lower levels of self reported physical activity, and to live alone than responders.

\section{Intervention delivery}

The dose and coverage of intervention delivery was high: $86.4 \%$ (999/1156) of the scheduled calls were delivered and $75.4 \%(218 / 289)$ of participants received all four calls. The average duration of calls was 39.2 minutes (SD 10.7) for the first call, then 23.8

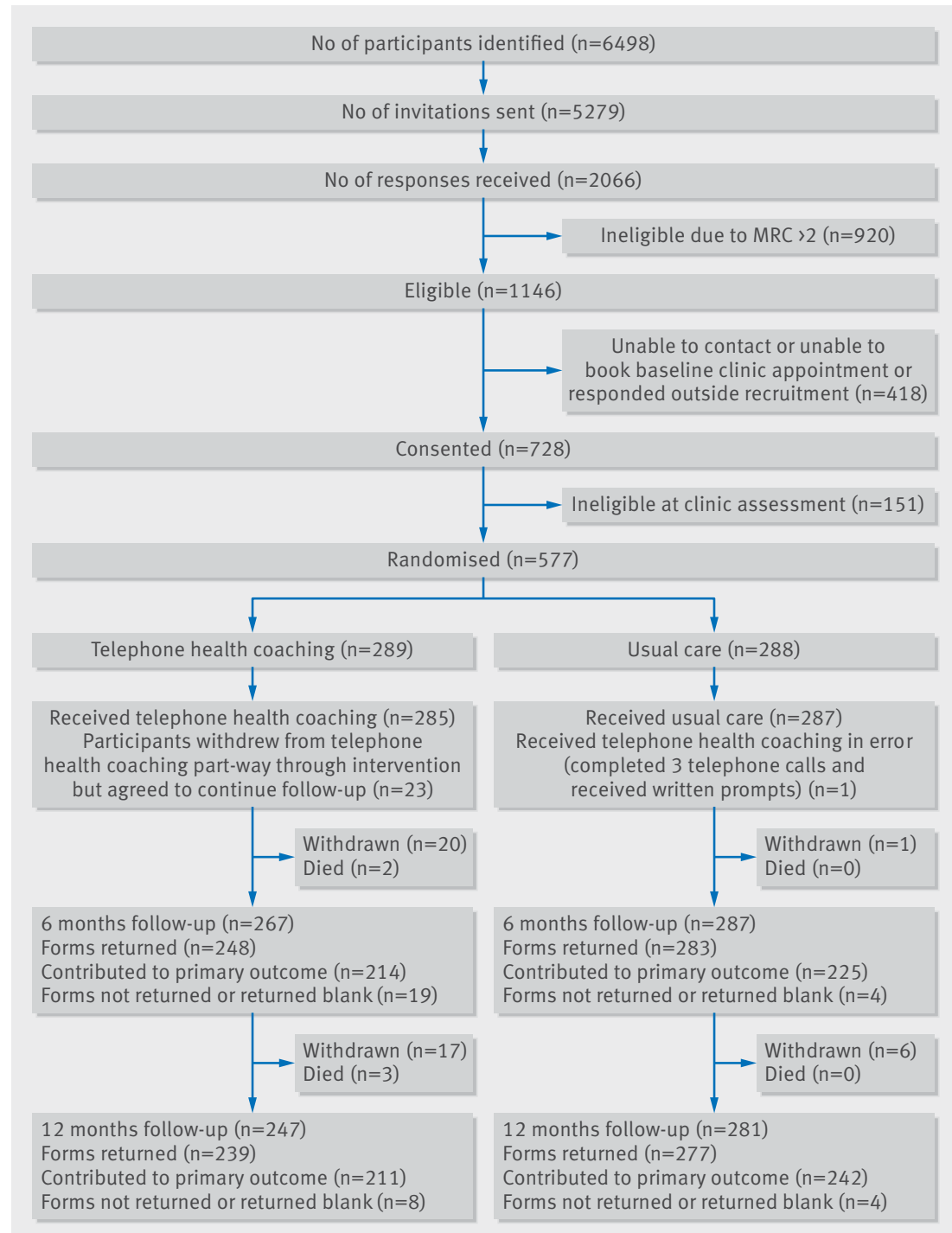

Fig 1 | Study flow diagram 


\begin{tabular}{|c|c|c|}
\hline Characteristic & Telephone health coaching $(n=289)$ & Usual care $(n=288)$ \\
\hline Mean (SD) age (years) & $70.7(8.8)$ & $70.2(7.8)$ \\
\hline Age $\geq 65$ years & $221(76)$ & $231(80)$ \\
\hline Men & $183(63)$ & $183(64)$ \\
\hline White race & $283(98)$ & 284 (99) \\
\hline $\begin{array}{l}\text { Median (interquartile range) age at completion of full time } \\
\text { education (years)* }\end{array}$ & $15(15-16)$ & $15(15-16)$ \\
\hline \multicolumn{3}{|l|}{ Highest level of qualification: } \\
\hline No formal qualification & $128(44)$ & $135(47)$ \\
\hline GCSE, CSE, O Level equivalent & $58(20)$ & $63(22)$ \\
\hline A Level, AS Level, or equivalent & $27(9)$ & $24(8)$ \\
\hline Degree level or higher & $35(12)$ & $41(14)$ \\
\hline Other & $40(14)$ & $23(8)$ \\
\hline Missing & $1(\measuredangle 1)$ & $2(1)$ \\
\hline Lives alone & $83(29)$ & $69(24)$ \\
\hline \multicolumn{3}{|l|}{ Employment statust: } \\
\hline Paid work & $58(20)$ & $53(18)$ \\
\hline Unemployed or looking for work & $3(1)$ & $5(2)$ \\
\hline Retired from paid work & $216(75)$ & $214(74)$ \\
\hline Looking after family or home & $8(3)$ & $9(3)$ \\
\hline Unable to work owing to health problems & $8(3)$ & $7(2)$ \\
\hline Other & $5(2)$ & $9(3)$ \\
\hline \multicolumn{3}{|l|}{ Clinical characteristics } \\
\hline Current smoker & $75(26)$ & $55(19)$ \\
\hline Mean (SD) body mass index $\left(\mathrm{kg} / \mathrm{m}^{2}\right)$ & $27.1(4.4)$ & $27.4(4.9)$ \\
\hline \multicolumn{3}{|l|}{ MRC dyspnoea scale score: } \\
\hline 1 & $89(31)$ & $76(26)$ \\
\hline 2 & $200(69)$ & $212(74)$ \\
\hline Mean (SD) FEV1 predicted (\%) & $71.2(18.9)$ & $72.1(18.7)$ \\
\hline \multicolumn{3}{|l|}{ FEV1 predicted (\%): } \\
\hline$<30$ & $1(<1)$ & $2(1)$ \\
\hline $30-49$ & $39(13)$ & $33(11)$ \\
\hline $50-79$ & $160(55)$ & $149(52)$ \\
\hline$\geq 80$ & $89(31)$ & $104(36)$ \\
\hline \multicolumn{3}{|l|}{ Comorbidities: } \\
\hline Cancer & $34(12)$ & $37(13)$ \\
\hline Diabetes & $32(11)$ & $36(13)$ \\
\hline High blood pressure & $135(47)$ & $123(43)$ \\
\hline Coronary heart disease & $34(12)$ & $44(15)$ \\
\hline Heart failure & $15(5)$ & $10(3)$ \\
\hline Stroke or mini-stroke & $16(6)$ & $25(9)$ \\
\hline Asthma & $98(34)$ & $100(35)$ \\
\hline Tuberculosis & $6(2)$ & $10(3)$ \\
\hline Osteoarthritis & $46(16)$ & $56(19)$ \\
\hline Rheumatoid arthritis & $22(8)$ & $25(9)$ \\
\hline Osteoporosis & $13(5)$ & $20(7)$ \\
\hline Depression & $44(15)$ & $57(20)$ \\
\hline Other condition & $37(13)$ & $52(18)$ \\
\hline
\end{tabular}




\begin{tabular}{|c|c|c|}
\hline Characteristic & Telephone health coaching $(n=289)$ & Usual care $(n=288)$ \\
\hline \multicolumn{3}{|l|}{ Medication taken regularly for lung problems: } \\
\hline Beta-2 agonist & $201(70)$ & $197(68)$ \\
\hline Inhaled steroid & $27(9)$ & $39(14)$ \\
\hline Atrovent or Spiriva & $109(38)$ & $117(41)$ \\
\hline Seretide & $88(30)$ & $92(32)$ \\
\hline Symbicort & $33(11)$ & $21(7)$ \\
\hline Theophylline or aminophylline tablets & $7(2)$ & $6(2)$ \\
\hline Steroid tablets & $5(2)$ & $9(3)$ \\
\hline Antibiotic or steroid course, or both, in past 12 months & $135(47)$ & $135(47)$ \\
\hline \multicolumn{3}{|l|}{ Health related quality of life } \\
\hline Mean (SD) SGRQ-C total score & $27.8(14.6)$ & $29.5(14.5)$ \\
\hline Mean (SD) SGRQ-C symptoms score & $48.5(21.7)$ & $47.9(20.7)$ \\
\hline Mean (SD) SGRQ-C activity score & $36.3(21.0)$ & $38.7(21.3)$ \\
\hline Mean (SD) SGRQ-C impact score & $15.4(13.4)$ & $17.6(13.9)$ \\
\hline Mean (SD) EuroQoL 5 Dimensions 5 Levels score & $0.90(0.13)$ & $0.89(0.13)$ \\
\hline \multicolumn{3}{|l|}{ Anxiety and depression $¥$} \\
\hline Mean (SD) anxiety subscale score & $3.8(3.4)$ & $4.3(3.8)$ \\
\hline Mean (SD) depression subscale score & $2.9(2.6)$ & $3.1(2.8)$ \\
\hline \multicolumn{3}{|l|}{ Physical activity } \\
\hline Mean (SD) minutes of MVPA/week by accelerometry & $372.1(305.1)$ & $379.1(282.9)$ \\
\hline Mean (SD) moderate MET minutes/week§ & $766.4(1253.9)$ & $941.5(1437.6)$ \\
\hline Mean (SD) vigorous MET minutes / week§ & $809.4(1771.5)$ & $910.2(1997.4)$ \\
\hline \multicolumn{3}{|l|}{ Self efficacy } \\
\hline Mean (SD) Stanford self efficacy score & $8.3(1.6)$ & $8.0(1.7)$ \\
\hline \multicolumn{3}{|c|}{$\begin{array}{l}\text { FEV1=forced expiratory volume in one second; SGRQ-C=St George's Respiratory Questionnaire; MVPA=moderate to vigorous physical activity; } \\
\text { MET=metabolic equivalent. } \\
\text { * One subject in the telephone health coaching group never went to school. } \\
\text { t Not mutually exclusive, participants could tick all that applied. } \\
\text { ₹ Using the Hospital Anxiety and Depression Scale. } \\
\text { § Using the International Physical Activity Questionnaire. }\end{array}$} \\
\hline
\end{tabular}

(9.2), 21.4 (8.6), and 20.6 minutes (8.7) for the second, third, and final calls respectively. Nurses briefly noted the content and duration of each telephone health coaching session. Most patients spoke to the same nurse for all four calls, although sometimes this was not possible owing to illness or leave. Smoking was discussed in $33 \%$ of sessions, physical activity in over $99 \%$, inhaler use technique in $90 \%$, and action planning in $88 \%$ of all calls. SMART (specific, measurable, achievable, relevant, and time bound) goals were set in $57 \%$ of calls for physical activity, in $11 \%$ for smoking cessation, and in $9 \%$ for inhaler use technique to be checked.

\section{Primary outcome}

At 12 months, there was no significant difference in the total SGRQ-C score (mean difference -1.3, 95\% confidence interval -3.6 to $0.9, \mathrm{P}=0.23$ ), although the direction favoured the intervention group. The mean difference in the SGRQ-C activity score was of borderline significance favouring the intervention group ( $-3.2,-6.3$ to $0.0, \mathrm{P}=0.05)$. Table 2 shows that there was no significant difference between groups for the SGRQ-C symptoms or impact scores.

\section{Secondary outcomes}

Table 2 shows that at six months, there were no significant differences in the SGRQ-C total and subscores. At six and 12 months, there were also no differences in the EuroQoL 5 Dimensions 5 Levels, Hospital Anxiety and Depression Scale, Stanford self efficacy scale, or level of breathlessness (MRC, table 3). At six months, total self reported physical activity, walking, moderate, and vigorous intensity activity were all significantly higher in the intervention arm (table 2). Differences favoured the intervention arm at 12 months, but they did not remain statistically significant. There was no difference in moderate or vigorous activity measured using accelerometry at 12 months. There was also no difference in smoking cessation rates at six and 12 months (table 3 ).

\section{Healthcare utilisation}

At six months, intervention participants reported lower doctor and pharmacist consultations, but higher all cause emergency department visits. There were no differences at 12 months (table 4). At six and 12 months, 106 (43\%) and 89 (37\%) of the intervention group respectively had been prescribed at least one 


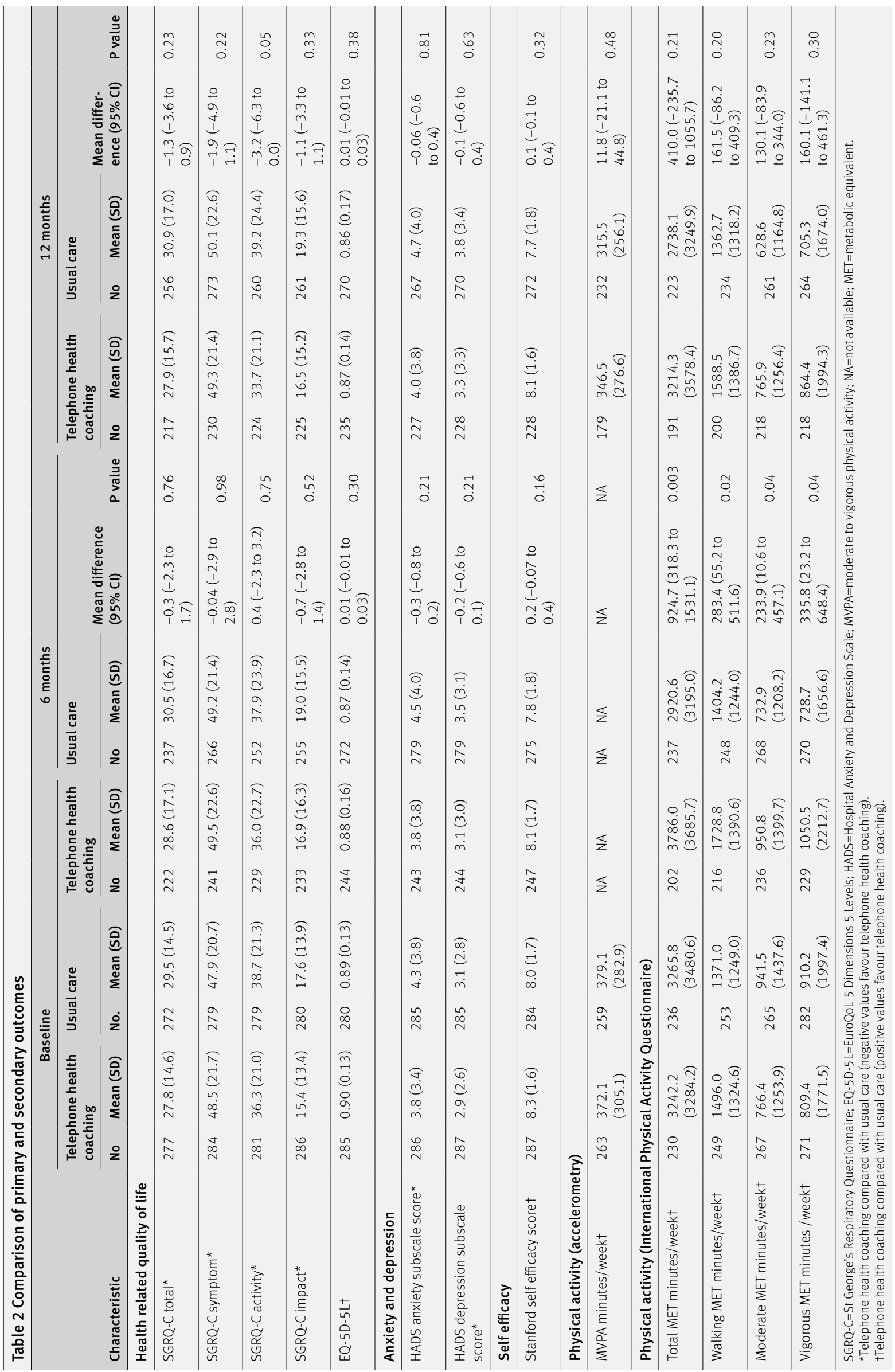

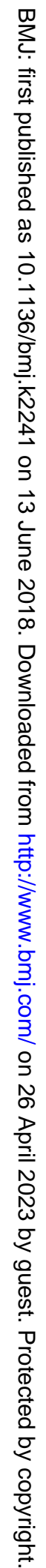




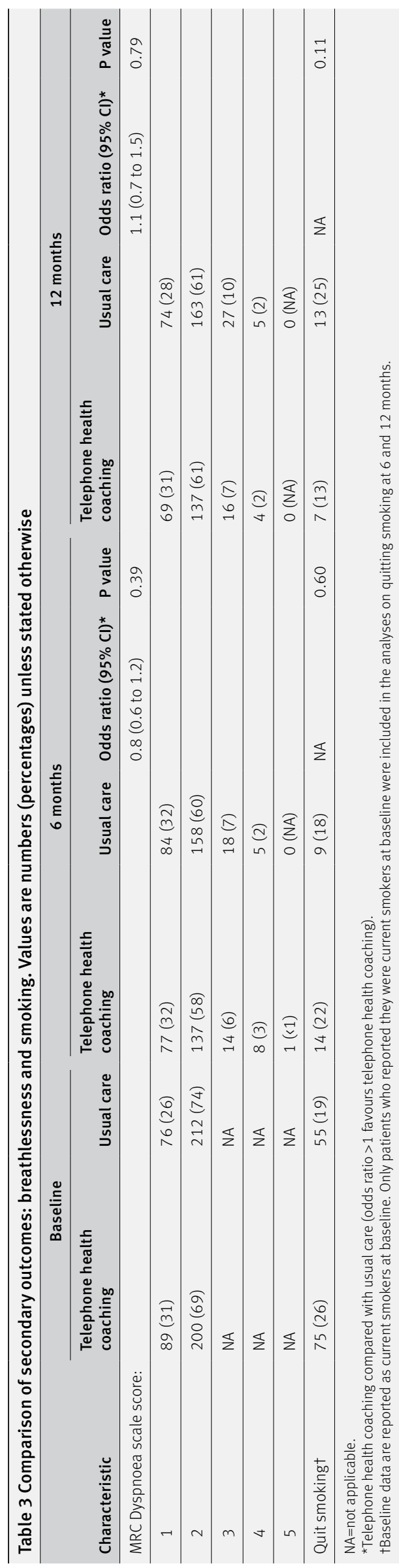

course of antibiotics compared with 105 (37\%) and 96 (35\%) of the usual care group (data not shown).

Activities targeted by telephone health coaching Physical activity and smoking cessation rates have been described previously. There was no difference between the groups in smoking quit attempts in the previous six months or attendance at smoking cessation services at either follow-up point. At six months, participants in the intervention group reported improved medication adherence, with significantly higher proportions having an inhaler check in the past six months (68\% v 55\%), an agreed care plan with a healthcare provider (44\% v 30\%), written advice about what to do if symptoms worsened ( $23 \% v 17 \%)$, and having an antibiotic rescue pack (37\% v 29\%). However, they did not report improved confidence in the use of rescue packs. Table 5 shows that many of these improvements were sustained at 12 months.

\section{Sensitivity and subgroup analyses}

There were no differences in the findings for the analysis in accordance with the protocol, when regression imputation was used to impute missing data, or when the analysis excluded questionnaires returned either early or late (see web appendix 3). Subgroup analyses also found no evidence that the effect size differed by age, sex, ethnicity, comorbidities, baseline MRC level, smoking status, Hospital Anxiety and Depression Scale, physical activity, predicted forced expiratory volume in one second ( $\geq 80$ or $<80$ ), degree of limitation of activities in the SGRQ-C, active engagers, or self efficacy. There was some evidence of an interaction with baseline SGRQ-C $(\mathrm{P}=0.04)$; with a greater benefit for intervention in participants with a baseline score $\geq 25$ (ie, those with poorer quality of life) (mean difference -3.0 , 95\% confidence interval -6.4 to 0.3$)$ compared with those with a baseline score $<25$ $(2.3,-1.6$ to 6.2$)$.

\section{Adverse events}

There were 44 serious adverse events reported by participants; 24 in the telephone health coaching arm and 20 in the usual care arm. Five deaths occurred in the intervention group due to cor pulmonale, stroke, ruptured aortic aneurysm, and malignancy (2). None were considered to be related to the intervention.

\section{Discussion \\ Principal findings}

This trial is the first to evaluate the effectiveness of a telephone health coaching intervention delivered by a nurse to support self management for patients with mild symptoms of chronic obstructive pulmonary disease (COPD). We showed an improvement in self reported self management activities in the telephone health coaching group compared with usual care, but we did not observe a difference in our primary outcome of health related quality of life measured by the St George's Respiratory Questionnaire (SGRQ-C) (nor in the impact, symptom, or activity domains) although 


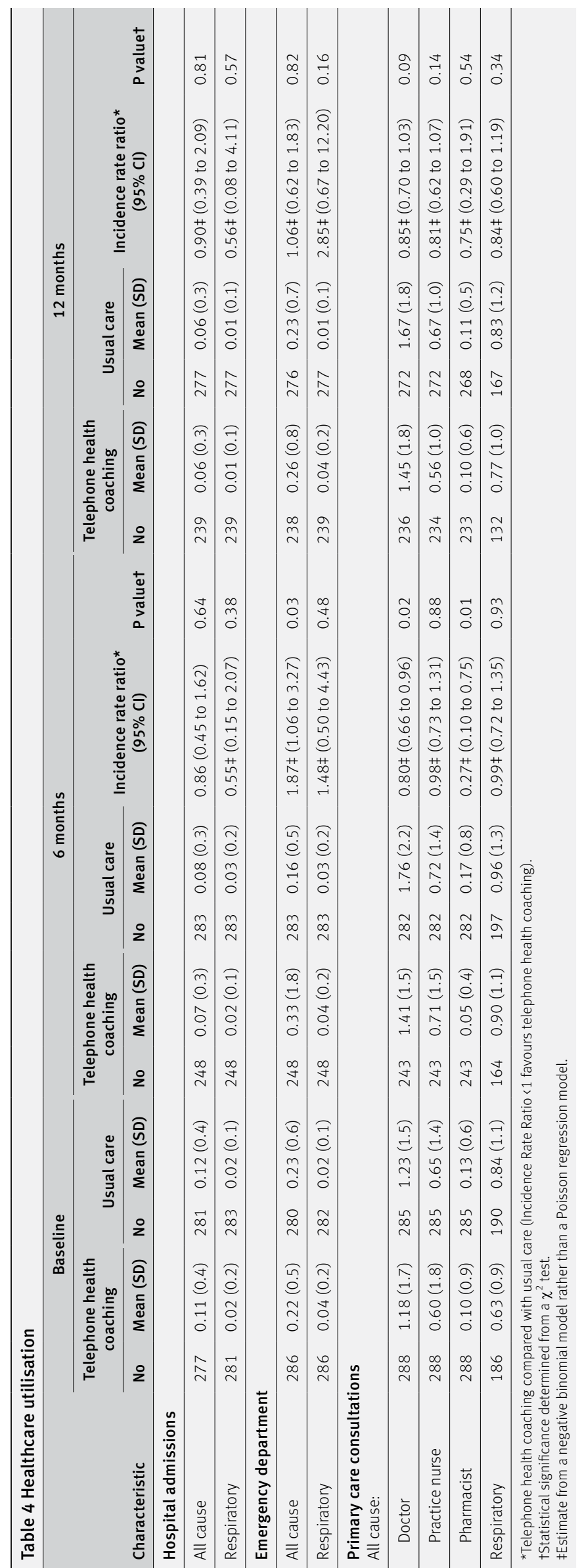

the confidence intervals did include the minimal clinically important difference of four points for the activity and symptom domains. Self reported physical activity was higher at six months in the intervention group, but this was not sustained at 12 months. In addition, activities targeted by the intervention, including patients asking a health care professional to check their inhaler use technique, asking their doctor to agree a care plan, and having a rescue pack were higher in the intervention group at six and 12 months follow-up, compared with usual care. This suggested that a proportion of intervention participants adopted active self management.

\section{Comparison with other studies}

Our approach was new in comparison to other trials of self management and telephone coaching interventions by targeting patients with mildly symptomatic disease. Most previous trials of COPD self management have excluded participants with Global Initiative for Obstructive Lung Disease (GOLD) stage I (mild airflow obstruction), ${ }^{940}$ whereas a third of our patients, were in this category, as we particularly wanted to evaluate an intervention for patients with mild COPD, who are a clinically important, but largely neglected group despite having a reduced health related quality of life. ${ }^{41}$

Systematic reviews of self management interventions have reported improvements in COPD related quality of life, measured by the SGRQ with a mean difference of -2.40 at 12 months follow-up. ${ }^{90} 42$ All reviews reported effects larger than the -1.3 points difference at 12 months found in our trial. Health related quality of life has been favoured as the main outcome for trials of COPD self management as functional status is important to patients and is sensitive to change, while lung function has a natural variation making it difficult to interpret change over short follow-up periods. Compared with other studies of self management in COPD, even those in milder populations, ${ }^{39}$ the SGRQ-C total score in our study was very low at baseline (representing a good health related quality of life). This potentially led to a floor effect, where change may be unlikely to be achievable, or improvement may only be observed over a much longer period. However, for the activity subscore of the SGRQ-C, the mean difference at 12 months (-3.2) found in our trial compares well with those of the systematic reviews, which report statistically significant mean differences of -2.75 and $-2.21 .^{940}$ These findings are consistent with the differences found at six months (at the end of the patient self management COPD intervention) in self reported physical activity (International Physical Activity Questionnaire). However, this result of reduced limitations to physical activities was not reflected in the self reported quantity or intensity of physical activity (International Physical Activity Questionnaire) or our objective measures of physical activity where there were no differences between groups at 12 months.

An Australian randomised controlled trial (RCT) of a 12 month intensive telephone health mentoring 


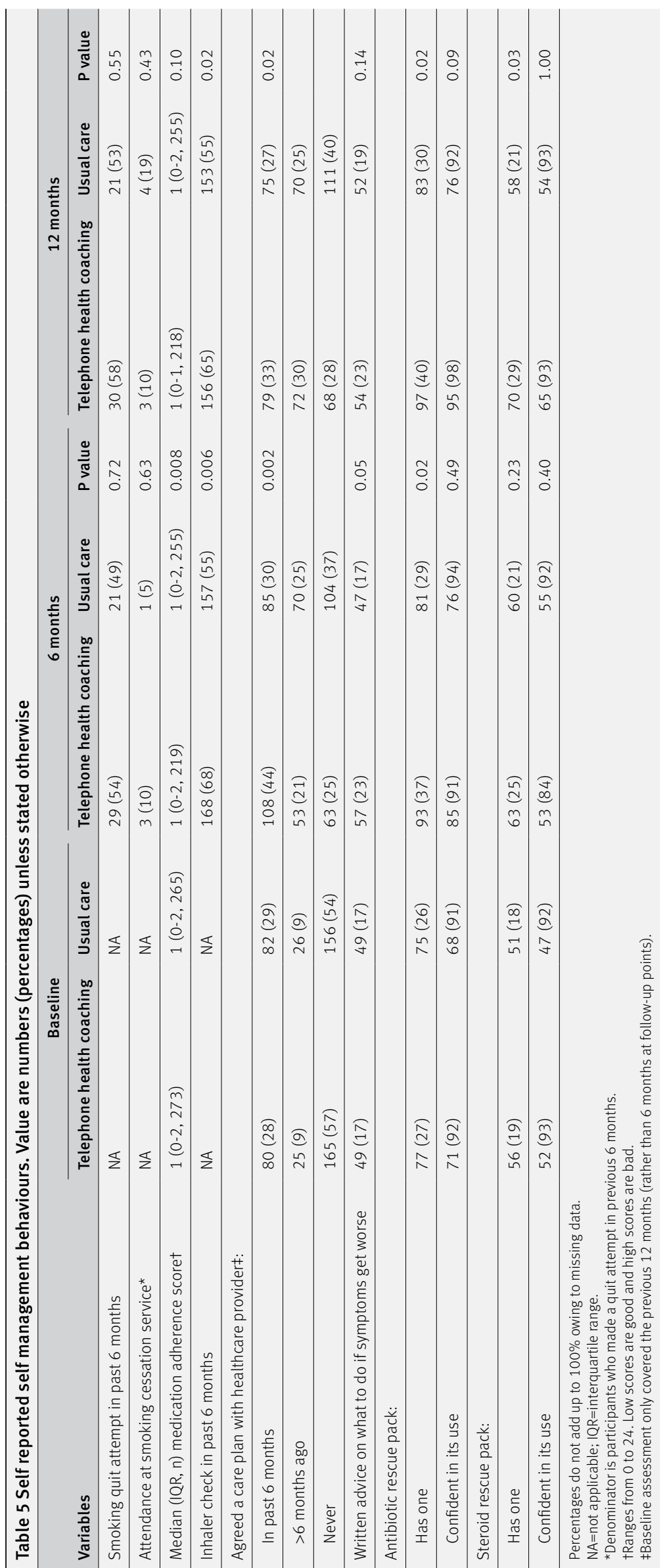

intervention for patients with moderate to severe COPD in primary care also did not report a difference in the SGRQ at 12 months; but did achieve greater improvements in self management capacity and in COPD knowledge than usual care. ${ }^{43}$ Similar to our trial, a 12 week RCT of an intensive automated telecoaching programme reported improvements in physical activity and the functional domain of the Clinical COPD Questionnaire, but not health related quality of life (COPD Assessment Test) at the end of the 12 week intervention period. ${ }^{44}$ Conversely, an RCT of telephone mentoring for home based walking showed no benefit in exercise capacity in patients with COPD before commencing a pulmonary rehabilitation programme, and also had a high withdrawal rate. ${ }^{45}$ In keeping with our findings, a rapid review of 30 RCTs of the effects of telephone health coaching to support self management of long term conditions reported improvements in health behaviours, but did not find conclusive evidence of improvements in quality of life. ${ }^{5}$

Observed short term improvements in self reported physical activity may have required a longer duration of support or intermittent maintenance activities to sustain changes. Primary care consultations were also lower in the intervention group at six months, which again may reflect the increased telephone contact in this period. A consistent message of the telephone health coaching was for intervention participants to use their routine appointments with primary care for their inhaler use technique to be checked or to discuss a care or action plan and it appears that participants heeded this message and did not book additional consultations for self management advice or support.

\section{Strengths and limitations of this study}

There were many strengths of this study. Firstly, focusing on a mildly symptomatic patient group who are largely excluded from other trials provided novelty and potential for clinical benefit. We used a multicentre study design incorporating a large sample of practices representative of the general UK population; a pragmatic design to accommodate a broad patient group with no selection by motivation to change health behaviours; spirometry was undertaken using trained staff and quality assured and we achieved a good follow-up rate. The intervention was underpinned by social cognitive theory and included techniques such as goal setting that have been shown to be effective in modifying behaviour and was at an intensity that might potentially be delivered in a publicly funded health service. ${ }^{46}$ We achieved good fidelity of delivery of the intervention, with $75 \%$ of intervention participants receiving all four calls and only four patients receiving none. There did not appear to be any contamination or change in behaviour in the usual care group with their self reported self management behaviours remaining static throughout the trial. In keeping with the pragmatic nature of this intervention, we did not check whether those who checked inhaler use technique had adequate training, but this is a core component of primary care management of COPD. ${ }^{24}$ 
Our study has some limitations. The intervention was in a group of people with only mild symptoms of breathlessness, who may not have considered themselves ill, thus a high degree of motivation may have been needed to take part. Highly motivated patients would be more likely to self manage their condition and change lifestyle behaviours. Our sample reported high levels of regular physical activity exceeding the lower recommended amount of moderate or vigorous activity per week at baseline; so, despite our efforts to recruit all eligible patients from primary care there is likely to have been self selection of people to the study, which may have affected capacity to improve, which is a feature of most behaviour change trials. The intervention did not meet the needs of some patients who withdrew from the intervention and in some cases also withdrew from the trial, resulting in an imbalance in follow-up rates between study arms. The patients who withdrew gave reasons including feeling that the intervention did not meet their needs as they were already physically active and some that were too unwell after an exacerbation. This may point to the need for more individual tailoring than actually occurred. In addition, delivery by telephone may give less opportunity for the building of rapport between the patient and nurse. Issues of rapport, acceptability, and tailoring of the intervention will be addressed in more detail in a separate publication of the qualitative evaluation. We did not observe large differences in the characteristics of those who withdrew from the trial, nor any differences in the interpretation of the primary outcome with a sensitivity analysis to assess the impact of missing data. The power calculation was based on detecting a four point difference in the SGRQ-C at 12 months (mean score 39, SD 15). Although participants in patient self management COPD had less severe disease at baseline (mean score 28.7) than expected, the standard deviation was 14.5 meaning we still have $80 \%$ power to detect the four point difference. However, owing to the lower SGRQ-C score at baseline, this four point difference now corresponds to a $14 \%$ proportional reduction compared with the $10 \%$ proportional reduction in the original sample size.

We trained the nurses for two days with further role plays and group calls to discuss challenges once the intervention had started. Our evaluation of the logs of their telephone calls and recorded calls identifies a variation in communication style from a patient centred to a more directive approach. Further, owing to the nature of recruitment across different sites, the distribution of calls completed by nurses was uneven. It was apparent that some patients were reluctant to set physical activity goals. It is possible that longer nurse training would have led to greater communication skills and more behavioural change, but this was a pragmatic study that aimed to evaluate an intervention that could be rolled out in practice. It is possible that a longer intervention duration, with calls beyond three months, would have led to greater effects and that in our group with predominantly mild disease, follow-up beyond 12 months might be needed to detect changes.
Implications for clinicians and policy makers

Adding telephone health coaching to support self management did not improve health related quality of life in our patient population with only mildly symptomatic disease and who were already quite physically active at baseline. It did, however, lead to an increase in self reported physical activity at six months, which is likely to result in health benefits, ${ }^{15} 16$ and self management activities which are likely to reduce the frequency and severity of exacerbations. While there is still uncertainty about best practice for managing people with mildly symptomatic disease, inhaled therapies are widely used in this group and improved engagement with education about correct delivery technique will help to realise improved outcomes for these patients. ${ }^{18} 19$ Self management support is currently recommended, but it is not likely to be well implemented. ${ }^{47}$ Much evidence for COPD self management support comes from patients recruited from secondary care and there needs to be a synthesis of the findings of support for self management in patients recruited from a primary care setting. It may be that among people with mildly symptomatic disease, self management support should be provided for those with poorest health related quality of life, which is the greatest predictor of future quality of life, ${ }^{48}$ or in those with the most frequent exacerbations. ${ }^{14}$ It may also be that a different health related quality of life outcome measure is needed for people with mild or early COPD that addresses limitations specific to the stage of their disease.

There is a lack of evidence of effective interventions for patients with mild COPD and this trial, while improving some self management behaviours, did not show evidence of clinical benefit. There remains a need to identify successful interventions for patients with milder symptoms of COPD and this also has clear implications for screening or case finding activities, which would identify patients with mild disease, and cannot be recommended while there is a lack of effective treatment options for this patient group. There are wider implications in the use of telephone health coaching; a rapid review reported that it appears to be most effective in vulnerable populations, who have difficulty accessing health services, ${ }^{4}$ which is not reflective of our study population. Supporting self management in patients with early disease, or risk factors, remains a challenge. Apart from diabetes prevention programmes, health services generally focus self management support and rehabilitation services on people with more advanced disease, but there is the potential for considerable health and health service gains if we could facilitate self management in patients with early disease and slow their decline. Establishing whether this is possible will require long term follow-up studies.

\section{Conclusions and policy implications}

A novel telephone health coaching intervention to promote behaviour change in patients with mild symptoms of dyspnoea in primary care led to changes in self management activities, but did not improve health related quality of life. There remains a clear 
need to identify risk mitigating interventions that can effectively prevent or delay disease progression in this patient group.

\section{AUTHOR AFFILIATIONS}

${ }^{1}$ Institute of Applied Health Research, Murray Learning Centre, University of Birmingham, Birmingham, B15 2ER, UK

${ }^{2}$ Department of Health Sciences, University of Leicester, Leicester, UK ${ }^{3}$ Birmingham Clinical Trials Unit, University of Birmingham, Birmingham, UK

${ }^{4}$ Department of Health Sciences, University of York, York, UK ${ }^{5}$ School of Sport, Exercise and Health Sciences, Loughborough University, Loughborough, UK

${ }^{6}$ Nuffield Department of Primary Care Health Sciences, University of Oxford, Oxford, UK

${ }^{7}$ Centre for Exercise and Rehabilitation Science, Biomedical Research Centre (Respiratory), University Hospitals of Leicester NHS Trust, Glenfield Hospital, Leicester UK

${ }^{8}$ Research Institute for Primary Care and Health Sciences, Keele University, Keele, UK

${ }^{9}$ School of Education Research, University of Birmingham, Birmingham, UK

${ }^{10}$ Royal Wolverhampton NHS Trust, New Cross Hospital, Wolverhampton, UK

${ }^{11}$ Warwick Primary Care, University of Warwick, Warwick, UK

We thank the patients, health care professionals, National Institute for Health Research Clinical Research Network, and the research nurses (Elizabeth Baker, Michael Loynd, Julie Young, Debbie D’Cruz, Karla Jennings-Preece, Alice Mackie, Sian Jones, and Caroline Reeves) who made this trial possible. We thank the Keele University Primary Care and Health Sciences for recruiting participants. We acknowledge the role of the Primary Care Clinical Research and Trials Unit, particularly Gurmail Rai, who developed the database and Andrea Roalfe who did the randomisation lists.

The Trial Steering Committee was chaired by Dr Hilary Pinnock (University of Edinburgh). Dr Rupert Jones (Plymouth University), Dr Jill Mollison (University of Oxford), and Mrs Maireade Bird (patient representative) were members

Contributors: KJ led the design of the trial, with contributions and advice from PAC, AD, RJ, CH, SS, PA, SJ, LD, and DF. KJ, RJ, PA, AD, and DF contributed to decisions on outcome measures. DF advised on involving practices. RJ and PA advised on lung function testing. $A D$ and $\mathrm{KJ}$ designed the intervention. SJ designed the economic evaluation. $\mathrm{KJ}, \mathrm{PAC}, \mathrm{CH}$, and Rhian Hughes (Keele University) were the local PIs. $\mathrm{CAH}$ and NI developed the statistical analysis plan and undertook the analyses. KJ drafted the paper with MSS, with critical input from all other authors. KJ and MSS contributed equally to this paper. All authors have read and approved the final draft. $\mathrm{KJ}$ is the guarantor. Funding: This paper summarises independent research funded by the National Institute for Health Research (NIHR) School for Primary Care Research and the NIHR Collaborations for Leadership in Applied Health Research and Care (CLAHRC) West Midlands. The views expressed are those of the authors and not necessarily those of the NHS, the NIHR, or the Department of Health and Social Care. SS is part funded by the CLAHRC East Midlands.

Competing interests: All authors have completed the ICMJE uniform disclosure form at www.icmje.org/coi_disclosure.pdf. RJ reports grants from National Institute for Health Research (NIHR) and PA reports grants from NIHR School for Primary Care Research during the conduct of the study. CH has received expenses and fees for his media work. $\mathrm{CH}$ has received expenses from the World Health Organization (WHO) and holds grant funding from the NIHR, the NIHR School of Primary Care Research, The Wellcome Trust, and the WHO. CH has received financial remuneration from an asbestos case. $\mathrm{CH}$ has also received income from the publication of a series of toolkit books published by Blackwells. On occasion, $\mathrm{CH}$ receives expenses for teaching evidencebased medicine and is also paid for his GP work in NHS out of hours. Centre for Evidence-Based Medicine jointly runs the EvidenceLive Conference with the BMJ and the Overdiagnosis Conference with some international partners which are based on a non-profit making model. Ethical approval: The study received ethical and research governance approval from the NHS Research Ethics Service, West Midlands, Solihul (Reference 13/WM/0206). All participants gave informed consent.
Data sharing: Data from the patient self management COPD study or the interventional materials are available from the corresponding author at c.b.jolly@bham.ac.uk. All the individual participant data collected during the trial (including the data dictionary) will be available, after deidentification, immediately after publication with no end date. The study protocol has been published. All proposals requesting data access will need to specify how it is planned to use the data, and all proposals will need approval of the trial coinvestigator team before data release.

Transparency: The guarantor (KJ) affirms that the manuscript is an honest, accurate, and transparent account of the study being reported; that no important aspects of the study have been omitted; and that any discrepancies from the study as planned (and, if relevant, registered) have been explained.

This is an Open Access article distributed in accordance with the terms of the Creative Commons Attribution (CC BY 4.0) license, which permits others to distribute, remix, adapt and build upon this work, for commercial use, provided the original work is properly cited. See: http://creativecommons.org/licenses/by/4.0/.

1 World Health Organisation. Noncommunicable disease: fact sheet: World Health Organisation; 2017 http://www.who.int/en/newsroom/fact-sheets/detail/chronic-obstructive-pulmonary-disease(copd)

2 Coulter A, Roberts S, Dixon A. Delivering better services for people with long term conditions. 2013. https://www.kingsfund.org.uk/ sites/default/files/field/field_publication_file/delivering-betterservices-for-people-with-long-term-conditions.pdf

3 Kiley I, Gibbons G. Developing a research agenda for primary prevention of chronic lung diseases--an NHLBI perspective. Am J Respir Crit Care Med 2014;189:762-3. doi:10.1164/rccm.201402O380ED

4 Lindner H, Menzies D, Kelly J, Taylor S, Shearer M. Coaching for behaviour change in chronic disease: a review of the literature and the implications for coaching as a self-management intervention. Aust J Prim Health 2003:9:177-85. doi:10.1071/PY03044.

5 Dennis SM, Harris M, Lloyd J, Powell Davies G, Faruqi N, Zwar N. Do people with existing chronic conditions benefit from telephone coaching? A rapid review. Aust Health Rev 2013;37:381-8.

6 GOLD. From the Global Strategy for the Diagnosis, Management and Prevention of COPD, Global Initiative for Chronic Obstructive Lung Disease. GOLD, 2017.

7 Mahon J, Bartlett C, Isojarvi I, Edwards M, Glanville J. York Health Consortium YH. Literature review: the economic costs of lung disease and the cost-effectiveness of policy and service interventions. 2017 Oct 2017. https://www. brit-thoracic.org uk/document-library/ research-and-innovation/documents/literature-review-the-economic costs-of-lung-disease/

8 Lenferink A, Brusse-Keizer M, van der Valk PD, et al. Selfmanagement interventions including action plans for exacerbations versus usual care in patients with chronic obstructive pulmonary disease. Cochrane Database Syst Rev 2017:8:CD011682. doi:10.1002/14651858.CD011682 pub2

9 Zwerink M, Brusse-Keizer M, van der Valk PD, et al. Self management for patients with chronic obstructive pulmonary disease. Cochrane Database Syst Rev 2014:3:CD002990

10 Adab P, Fitzmaurice DA, Dickens AP, et al. Cohort Profile: The Birmingham Chronic Obstructive Pulmonary Disease (COPD) Cohort Study. Int J Epidemiol 2017;46:23.

11 Haughney J, Gruffydd-Jones K, Roberts J, Lee AJ, Hardwell A McGarvey L. The distribution of COPD in UK general practice using the new GOLD classification. Eur Respir / 2014;43:993-1002. doi:10.1183/09031936.00065013

12 Jordan RE, Adab P, Sitch A, et al. Targeted case finding for chronic obstructive pulmonary disease versus routine practice in primary care (TargetCOPD): a cluster-randomised controlled trial. Lancet Respir Med 2016:4:720-30 doi:10.1016/S2213-2600(16)30149-7

13 Anthonisen NR, Connett JE, Kiley JP, et al. Effects of smoking intervention and the use of an inhaled anticholinergic bronchodilator on the rate of decline of FEV1. The Lung Health Study. JAMA 1994;272:1497-505. doi:10.1001/ jama.1994.03520190043033

14 Dransfield MT, Kunisaki KM, Strand MJ, et al, COPDGene Investigators. Acute Exacerbations and Lung Function Loss in Smokers with and without Chronic Obstructive Pulmonary Disease. Am J Respir Crit Care Med 2017:195:324-30.

15 Gimeno-Santos E, Frei A, Steurer-Stey C, et al, PROactive consortium. Determinants and outcomes of physical activity in patients with COPD: a systematic review. Thorax 2014;69:731-9. doi:10.1136/ thoraxinl-2013-204763

16 Vaes AW, Garcia-Aymerich J, Marott JL, et al. Changes in physical activity and all-cause mortality in COPD. Eur Respir / 2014:44:1199209. doi:10.1183/09031936.00023214 
17 Watz H, Waschki B, Meyer T, Magnussen H. Physical activity in patients with COPD. Eur Respir / 2009;33:262-72. doi:10.1183/09031936.00024608

18 Vogelmeier C, Fabbri LM, Rabe KF, et al. Effect of tiotropium vs. salmeterol on exacerbations: GOLD II and maintenance therapy naïve patients. Respir Med 2013;107:75-83. doi:10.1016/j. rmed.2012.09.015

19 Zhou Y, Zhong NS, Li X, et al. Tiotropium in Early-Stage Chronic Obstructive Pulmonary Disease. N Engl / Med 2017;377:923-35. doi:10.1056/NEJMoa1700228

20 Sanchis J, Gich I, Pedersen SAerosol Drug Management Improvement Team (ADMIT). Systematic Review of Errors in Inhaler Use: Has Patient Technique Improved Over Time?Chest 2016;150:394-406. doi:10.1016/j.chest.2016.03.041

21 Sidhu MS, Daley A, Jordan R, et al. Patient self-management in primary care patients with mild COPD - protocol of a randomised controlled trial of telephone health coaching. BMC Pulm Med 2015;15:16. doi:10.1186/s12890-015-0011-5

22 Boutron I, Altman DG, Moher D, Schulz KF, Ravaud PCONSORT NP Group. Consort statement for randomized trials of nonpharmacologic treatments: A 2017 update and a consort extension for nonpharmacologic trial abstracts. Ann Intern Med 2017;167:40-7. doi:10.7326/M17-0046

23 Rick J, Graffy J, Knapp P, et al. Systematic techniques for assisting recruitment to trials (START): study protocol for embedded, randomized controlled trials. Trials 2014;15:407. doi:10.1186/1745-6215-15-407

24 NICE. Chronic obstructive pulmonary disease in over 16s: diagnosis and management [CG101]. London 2010. https://www.nice.org.uk/ guidance/cg101

25 Patient. Chronic Obstructive Pulmonary Disease: Patient Platform Ltd; 2014 https://patient.info/doctor/chronic-obstructive-pulmonarydisease-pro

26 Bandura A. Self-efficacy: toward a unifying theory of behavioral change. Psychol Rev 1977;84:191-215. doi:10.1037/0033295X.84.2.191

27 Lorig KR, Holman H. Self-management education: history, definition, outcomes, and mechanisms. Ann Behav Med 2003;26:1-7. doi:10.1207/S15324796ABM2601 01

28 Lorig KR, Sobel DS, Ritter PL, Laurent D, Hobbs M. Effect of a selfmanagement program on patients with chronic disease. Eff Clin Pract 2001;4:256-62.

29 Bravata DM, Smith-Spangler C, Sundaram V, et al. using pedometers to increase physical activity and improve health: a systematic review. JAMA 2007;298:2296-304. doi:10.1001/jama.298.19.2296

30 Eakin EG, Lawler SP, Vandelanotte C, Owen N. Telephone interventions for physical activity and dietary behavior change: a systematic review. Am J Prev Med 2007;32:419-34. doi:10.1016/j. amepre.2007.01.004

31 Kahn EB, Ramsey LT, Brownson RC, et al. The effectiveness of interventions to increase physical activity. A systematic review. Am J Prev Med 2002;22(Suppl):73-107. doi:10.1016/S07493797(02)00434-8

32 Ogilvie D, Foster CE, Rothnie H, et al, Scottish Physical Activity Research Collaboration. Interventions to promote walking: systematic review. BMJ 2007:334:1204. doi:10.1136/bmj.39198.722720.BE

33 Jones PW, Quirk FH, Baveystock CM, Littlejohns P. A selfcomplete measure of health status for chronic airflow limitation. The St. George's Respiratory Questionnaire. Am Rev Respir Dis 1992;145:1321-7. doi:10.1164/ajrccm/145.6.1321

34 Fletcher CM. The clinical diagnosis of pulmonary emphysema; an experimental study. Proc R Soc Med 1952;45:577-84.

35 Craig CL, Marshall AL, Sjöström M, et al. International physical activity questionnaire: 12 -country reliability and validity.
Med Sci Sports Exerc 2003;35:1381-95. doi:10.1249/01. MSS.0000078924.61453.FB

36 Zigmond AS, Snaith RP. The hospital anxiety and depression scale. Acta Psychiatr Scand 1983;67:361-70. doi:10.1111/j.1600-0447.1983.tb09716.x

37 Herdman M, Gudex C, Lloyd A, et al. Development and preliminary testing of the new five-level version of EQ-5D (EQ-5D-5L). Qual Life Res 2011;20:1727-36. doi:10.1007/s11136-011-9903-x

38 Jones PW. George's respiratory questionnaire: MCID. COPD 2005:2:75-9. doi:10.1081/COPD-200050513

39 van Wetering CR, Hoogendoorn M, Mol SJ, Rutten-van Mölken MP, Schols AM. Short- and long-term efficacy of a communitybased COPD management programme in less advanced COPD: a randomised controlled trial. Thorax 2010;65:7-13. doi:10.1136/ thx.2009.118620

40 Cannon D, Buys N, Sriram KB, Sharma S, Morris N, Sun J. The effects of chronic obstructive pulmonary disease self-management interventions on improvement of quality of life in COPD patients: A meta-analysis. Respir Med 2016;121:81-90. doi:10.1016/j. rmed.2016.11.005

41 Wacker ME, Jörres RA, Karch A, et al, COSYCONET study group. Relative impact of COPD and comorbidities on generic healthrelated quality of life: a pooled analysis of the COSYCONET patient cohort and control subjects from the KORA and SHIP studies. Respir Res 2016;17:81. doi:10.1186/s12931-016-0401-0

42 Jolly K, Majothi S, Sitch AJ, et al. Self-management of health care behaviors for COPD: a systematic review and meta-analysis. Int J Chron Obstruct Pulmon Dis 2016;11:305-26. doi:10.2147/COPD. S90812

43 Walters J, Cameron-Tucker H, Wills K, et al. Effects of telephone health mentoring in community-recruited chronic obstructive pulmonary disease on self-management capacity, quality of life and psychological morbidity: a randomised controlled trial. BM Open 2013;3:e003097. doi:10.1136/bmjopen-2013-003097

44 Demeyer H, Louvaris Z, Frei A, et al, Mr Papp PROactive study group and the PROactive consortium. Physical activity is increased by a 12 -week semiautomated telecoaching programme in patients with COPD: a multicentre randomised controlled trial. Thorax 2017;72:415-23. doi:10.1136/thoraxinl-2016-209026

45 Cameron-Tucker HL, Wood-Baker R, Joseph L, Walters JA, Schüz N, Walters $\mathrm{EH}$. A randomized controlled trial of telephone-mentoring with home-based walking preceding rehabilitation in COPD. Int J Chron Obstruct Pulmon Dis 2016;11:1991-2000. doi:10.2147/ COPD.S109820

46 NICE. Behaviour change: general approaches. Public Health Guideline PH6. NICE, London 2007. https://www.nice.org.uk/Guidance/ph6

47 Khan A, Dickens AP, Adab P, Jordan RE. Self-management behaviour and support among primary care COPD patients: cross-sectional analysis of data from the Birmingham Chronic Obstructive Pulmonary Disease Cohort. NPJ Prim Care Respir Med 2017;27:46. doi:10.1038/s41533-017-0046-6

48 Siebeling L, Musoro JZ, Geskus RB, et al. Prediction of COPD-specific health-related quality of life in primary care COPD patients: a prospective cohort study. NPJ Prim Care Respir Med 2014;24:14060 doi:10.1038/npjpcrm.2014.60

Supplementary materials: Outline of the intervention components

Supplementary materials: Accelerometry methods and adherence

Supplementary materials: Results of sensitivity analyses for the PSM-COPD study 